\title{
Promoter hypomethylation regulates CD133 expression in human gliomas
}

Kouichi Tabu ${ }^{1}$, Ken Sasai ${ }^{1}$, Taichi Kimura ${ }^{1}$, Lei Wang ${ }^{1}$, Eiko Aoyanagi ${ }^{1}$, Shinji Kohsaka ${ }^{1}$, Mishie Tanino ${ }^{1}$, Hiroshi Nishihara ${ }^{1}$, Shinya Tanaka ${ }^{1}$

${ }^{1}$ Laboratory of Molecular and Cellular Pathology, Hokkaido University Graduate School of Medicine, N15, W7, Kita-ku, Sapporo 060-8638, Japan

Brain tumor-initiating cells (BTICs) have been enriched using antibodies against the cell surface protein CD133; however, the biological relevance and the regulatory mechanism of CD133 expression in human gliomas are not yet understood. In this study, we initially demonstrated that CD133 was overexpressed in high-grade human glioblastomas where CD133-positive cells were focally observed as a micro-cluster. In addition, CD133 transcripts with exon 1A, 1B, or $1 \mathrm{C}$ were predominantly expressed in glioblastomas. To elucidate the mechanism regulating this aberrant expression of CD133, three proximal promoters (P1, P2, and P3) containing a CpG island were isolated. In U251MG and T98G glioblastoma cells, the $P 1$ region flanking exon $1 \mathrm{~A}$ exhibited the highest activity among the three promoters, and this activity was significantly inactivated by in vitro methylation. After treatment with the demethylating agent 5 -azacytidine and/or the histone deacetylase inhibitor valproic acid, the expression level of CD133 mRNA was significantly restored in glioma cells. Importantly, hypomethylation of $\mathrm{CpG}$ sites within the P1, P2, and P3 regions was observed by bisulfite sequencing in human glioblastoma tissues with abundant CD133 mRNA. Taken together, our results indicate that DNA hypomethylation is an important determinant of CD133 expression in glioblastomas, and this epigenetic event may be associated with the development of BTICs expressing CD133.

Keywords: glioma, CD133, promoter, hypomethylation

Cell Research (2008) 18:1037-1046. doi: 10.1038/cr.2008.270; published online 5 August 2008

\section{Introduction}

Gliomas are the most common primary tumors arising in the human central nervous system, and these tumors are classified into grades I, II, III, or IV using the WHO classification system [1]. Despite recent advances in the combination treatment of surgery together with chemo- and radiotherapies, the prognosis of malignant glioma is still extremely poor. For example, the median survival rate of glioblastoma patients with a grade IV tumor is less than one year from the initial diagnosis [2]. Thus, a complete understanding of the properties of malignant glioma cells and the development of new therapies for this disease are urgently needed.

Recently, substantial evidence has been accumulating

\footnotetext{
Correspondence: Shinya Tanaka

Tel: +81 11706 5053; Fax: +81 117065902

E-mail: tanaka@med.hokudai.ac.jp

Received 19 February 2008; revised 21 April 2008, accepted 9 May 2008; published online 5 August 2008
}

regarding brain tumors as well as other malignancies, and these data indicate that a small population with stem celllike properties, referred to as 'cancer stem cells', [3] are primarily involved in tumor formation and maintenance [4-11]. Brain tumor-initiating cells (BTICs) have been enriched by looking for expression of the neural stem cell (NSC) marker CD133, also known as prominin-1 or $\mathrm{AC} 133[4,5,12,13]$. CD133 ${ }^{+}$tumor cells can initiate neurospheres, which exhibit self-renewal, differentiation, and proliferation resembling that of normal NSCs [4]. The transplantation of $\mathrm{CD} 133^{+}$tumor cells, but not CD133- cells, into NOD/SCID mice is sufficient to produce tumors phenotypically identical to the patient's original tumors [5], although in some cases the CD133- cells are also tumorigenic [14]. CD133+ tumor cells were also reported to possess enhanced chemoresistance and radioresistance, resulting in the regulation of tumor progression and recurrence [15-17]. Therefore, $\mathrm{CD} 133^{+}$tumor cells are thought to be a potential therapeutic target for eradicating human brain tumors. Furthermore, a recent analysis revealed that expression of CD133 was much higher in recurring glio- 
blastoma tumors than in the primary lesion [18], suggesting that the regulatory mechanisms of CD133 expression could help elucidate the development of gliomas.

CD133 is a cellular surface glycoprotein containing five transmembrane regions and two glycosylated extracellular loops with a molecular weight of $97-120 \mathrm{kDa}$ [19]. CD133 was found localized in microvilli and protruding plasma membrane, thus interacting with membrane cholesterol [20]. The $C D 133$ gene is also known to possess five promoters yielding different spliced transcripts and five alternative first exons that are expressed in a tissue-specific manner [21]. However, the mechanisms of CD133 expression and its regulation remain largely unclear.

Changes in DNA methylation patterns are an important hallmark of tumor development and progression [22]. Although the role of hypermethylation in the silencing of tumor suppressor genes is now well-documented [23], a reduction in the level of methylation also contributes to neoplastic progression in numerous types of human cancer, including glioblastomas [24,25].

In this study, we assessed the expression of CD133 in 22 human gliomas of various grades and demonstrated that the aberrant expression of CD133 is restricted in human glioblastomas with a higher clinicopathological grade. To elucidate the mechanisms underlying the aberrant expression of CD133 in glioblastoma cells, three CD133 promoters, including a $\mathrm{CpG}$ island, were analyzed. Using a panel of glioblastoma cell lines and tissue samples, we show that the transcription of CD133 in glioblastoma is controlled by DNA hypomethylation-dependent mechanisms.

\section{Results}

Overexpression of CD133 mRNA in human glioblastoma specimens

It has been previously reported that CD133 is expressed in glioblastoma tissues $[4,5]$, but its expression in other types of glial tumors has not yet been examined. To characterize the expression of CD133 in different types of gliomas, we first analyzed the expression of CD133 mRNA in eight cases of low-grade glioma (grades I-III) and 14 cases of glioblastoma (grade IV) (Supplementary information, Table S1). As presented in Figure 1A panel-i, the expression of CD133 mRNA was minimal in low grade gliomas (grades I and II), which are similar to normal brain tissue. Even in a grade III tumor, only one of the four samples showed expression of CD133 (T7). In contrast, higher levels of CD133 were observed in 10 of 14 (71\%) grade IV glioblastomas. These data suggest that the level of CD133 mRNA is frequently increased in human glioblastoma tissues and is correlated with glioma grading.

To confirm these data, immunohistochemical analysis of CD133 protein expression was performed using corresponding glioma tissues, and, in most cases, we determined that the CD133 immunopositivity of glioblastomas correlated with the RT-PCR results (Figure 1B and Supplementary information, Figure S1). Glioblastomas exhibited various histopathological phenotypes and two patterns of CD133 staining, shown as pattern 1 (broad) or pattern 2 (scattered). Positive staining for CD133 was predominantly observed in the cytoplasm of glioblastoma cells. We also compared the immunopositivities of CD133 and nestin, as nestin is another NSC marker. We found that CD133 is only expressed in a subset of nestin-positive cells, with some variation $(30 \%$ of nestin-positive cells expressed CD133 in pattern 1, and less than $3 \%$ of nestin-positive cells were CD133-positive in pattern 2, as shown in Figure 1B). Furthermore, CD133-positive cells were found to form micro-clusters in the specimens (Figure 1C).

The human CD133 gene has also been reported to produce at least two differentially spliced ORF isoforms, CD133s2 (2 $598 \mathrm{bp}$ ) and CD133s1 (2 $571 \mathrm{bp}$ ). The latter lacks exon 3 (composed of 27 nucleotides) of the CD133 gene [26]. To determine the relationship between these two ORF isoforms and glioma grading, we performed RT-PCR analysis with specific primers flanking the deleted sequences. RT-PCR analysis for splicing variants showed that five cases of glioblastomas overexpressed both CD133s1 and CD133s2 (T12, 13, 14, 18, and 19), three cases predominantly exhibited CD133s1 (T9, 17, and 21), and the other two cases predominantly exhibited CD133s2 (T11 and 20). The presence of CD133s1, but not CD133s2, was detectable in normal brain tissues (Figure 1A panel-ii), suggesting that CD133s2 might be an important indicator of glioma malignancy.

\section{5'-UTR pattern of CD133 transcripts in human glioma specimens}

Restricted expression of CD133 in glioblastomas prompted us to investigate the regulation of CD133 expression in relation to glioma development. Thus, we analyzed the promoter regions of the $C D 133$ gene, which contains at least 29 exons. The translational initiation site is positioned in exon 2 , and exon 1 produces alternatively spliced $5^{\prime}$-untranslated regions (5'-UTRs), designated as exons 1A, $1 \mathrm{~B}$, 1C, 1D1, 1D2, 1D3, 1E1, 1E2, 1E3, and 1E4. Transcription at these sites is controlled by five alternative promoters known as P1, P2, P3, P4, and P5 (Figure 2A upper scheme) [21]. To determine the first exon transcribed in glioma cells, we utilized RT-PCR to investigate the expression pattern of CD133 exon 1s in 22 glioma specimens (Figure 1A panel-iv-viii). In all analyzed samples including normal brain, the exon 1A- and 1B-containing single band was predominantly detected, which correlates with the level of 
A

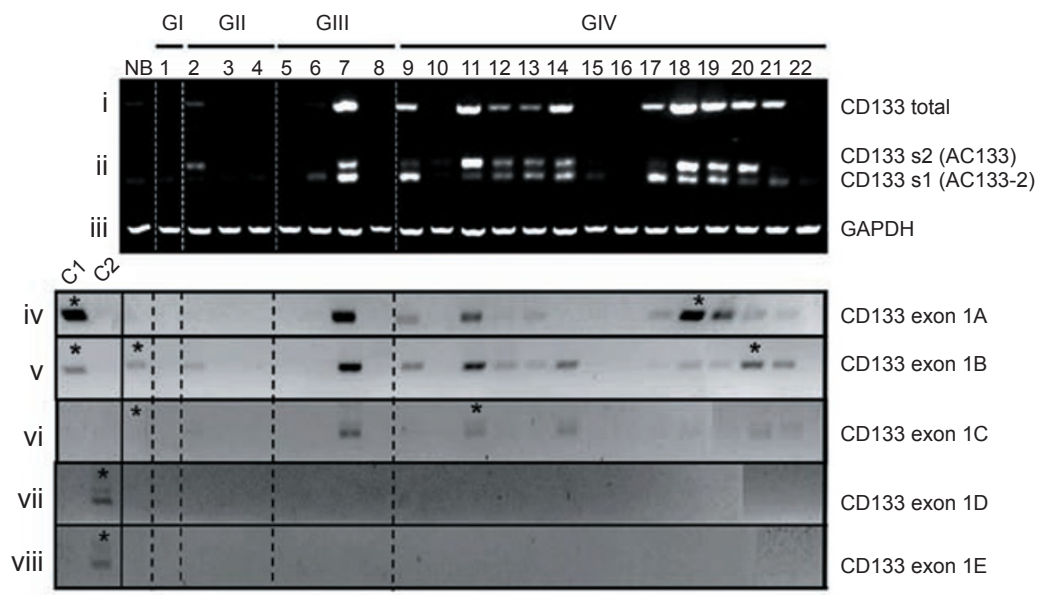

B
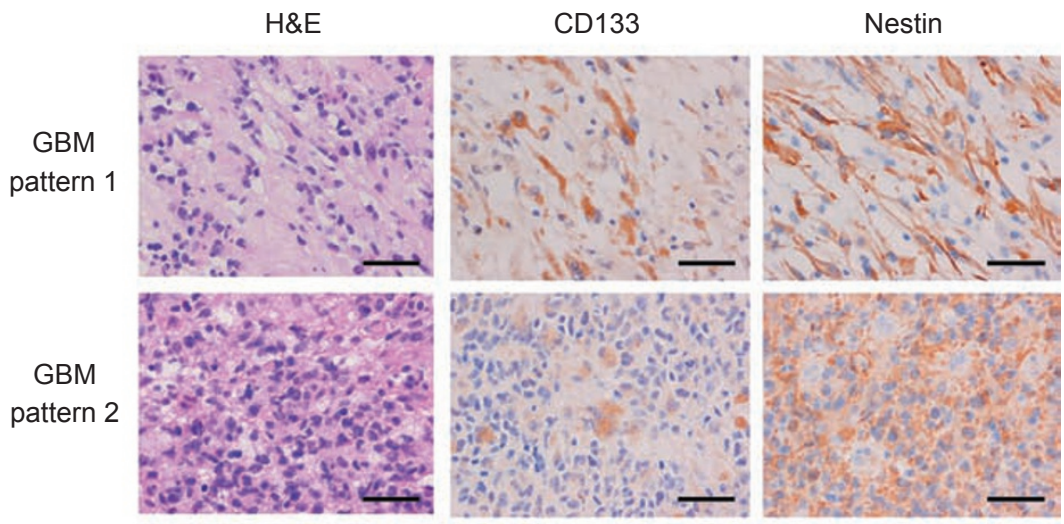

C
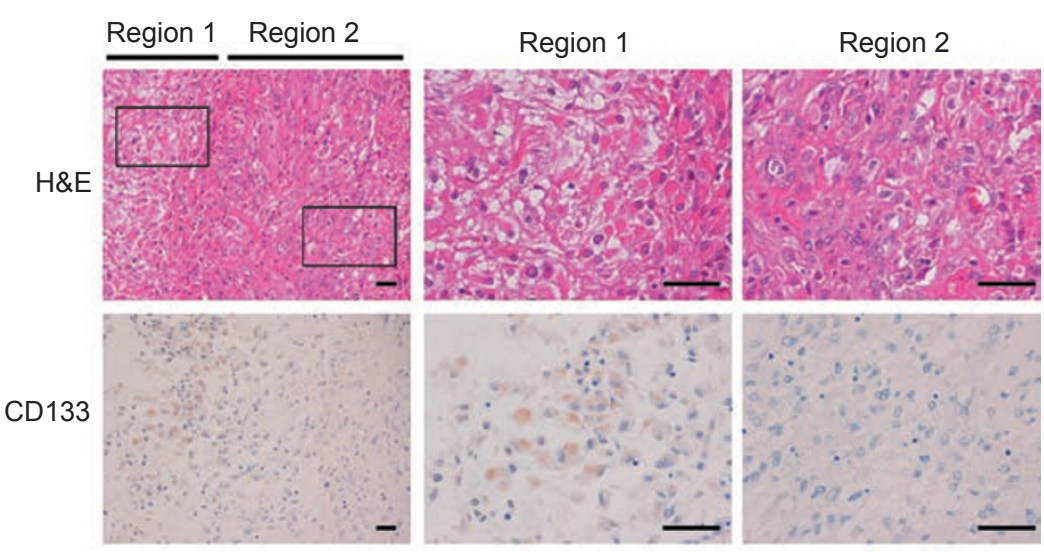

Figure 1 Increased expression of CD133 mRNA containing exons 1A, 1B, and 1C in human glioblastoma tissues. (A) (i-iii) RTPCR analysis of CD133 mRNA expression in various grades of human glioma tissues. (i) Expression of total CD133 mRNA. The number of PCR cycles was 30. (ii) Expression of two CD133 N-terminal splice variants within the ORF. (iii) GAPDH as an internal control. NB, normal brain tissue. (iv-viii) PCR analysis of expression patterns for CD133 5' -UTR isoforms containing exons $1 \mathrm{~A}$ (iv), 1B (v), 1C (vi), 1D (vii), and 1E (viii) in human glioma tissues. PCR amplification was performed using five forward primers located in exons 1A, 1B, 1C, 1D1, and 1E1 and a reverse primer located in the common exon 2 of the CD133 gene, as shown in Figure 2A. CDNA from normal human kidney (C1) and testis (C2) tissues were used as positive controls for exons $\mathrm{A}, \mathrm{B}$, and $\mathrm{C}$ and exons $\mathrm{D}$ and $\mathrm{E}$, respectively. Portions of fragments from kidney, testis, and gliomas T11, T18, and T20 (indicated by asterisks) were ligated into PCR Blunt II Topo and sequenced to confirm that they were the expected CD133 transcripts. (B) IHC of glioblastoma tissues with an anti-CD133 antibody. The representative results of two patterns of staining for T18 are displayed. Bar=100 $\mu \mathrm{m}$. (C) Focal localization of CD133-positive cells detected by IHC. A micro-cluster of CD133 was observed and called region 1 . The majority of CD133-negative cells were labeled as region 2 . The representative results of staining $\mathrm{T} 10$ are displayed. Bar=100 $\mu \mathrm{m}$. 
CD133 expression (Figure 1A panel-iv and -v). It should be noted that the exon $1 \mathrm{C}$-containing isoforms, which have previously been reported as undetectable in any tissues [21], were detected as multiple bands in the kidney, normal brain, and some glioma tissues (Figure 1A panel-vi). However, exon 1D- and 1E-containing bands were observed only in the testis as a positive control, even when a high PCR cycle number ( $>40$ cycles) was used for amplification (Figure 1A panel-vii and -viii). All of the RT-PCR products were verified by DNA sequencing to contain the sequences corresponding to each exon 1 isoform. Furthermore, we identified three novel exons, designated exons C2, D4, and E5, and five elongated forms of the exons (Figure 2A lower scheme and Supplementary information, Table S2). All of the newly discovered exon-intron junctions conform to the GT-AG consensus rule (Supplementary information, Table S3), and all splicing patterns found in this study are described (Supplementary information, Figure
$\mathrm{S} 2$ ). Because our results suggested that the $C D 133$ gene is transcribed from any or all of $\mathrm{P} 1, \mathrm{P} 2$, and $\mathrm{P} 3$ located in the 5 '-flanking region of exon $1 \mathrm{~A}, 1 \mathrm{~B}$, and $1 \mathrm{C}$, in human glioma cells, we focused on these three promoters in the following experiments.

\section{Activity of proximal promoters of CD133 in human glio-} blastoma cells

Reporter gene constructs were designed to determine the essential promoter for CD133 exon 1A-, 1B-, and $1 \mathrm{C}$-derived transcripts. The three promoter constructs, pGL3enh-P1, P2, and P3, each had the appropriate genomic DNA sequence (upstream of exons $1 \mathrm{~A}, 1 \mathrm{~B}$, or $1 \mathrm{C}$, as well as the first $10 \mathrm{bp}$ of each exon) fused ahead of the firefly luciferase cDNA in the pGL3 enhancer vector (Figure 2B left scheme). A reporter assay was performed in U251MG and $\mathrm{T} 98 \mathrm{G}$ glioblastoma cells, and remarkable activation of P1 (about 24-fold for U251MG and 14-fold for T98G) was

A

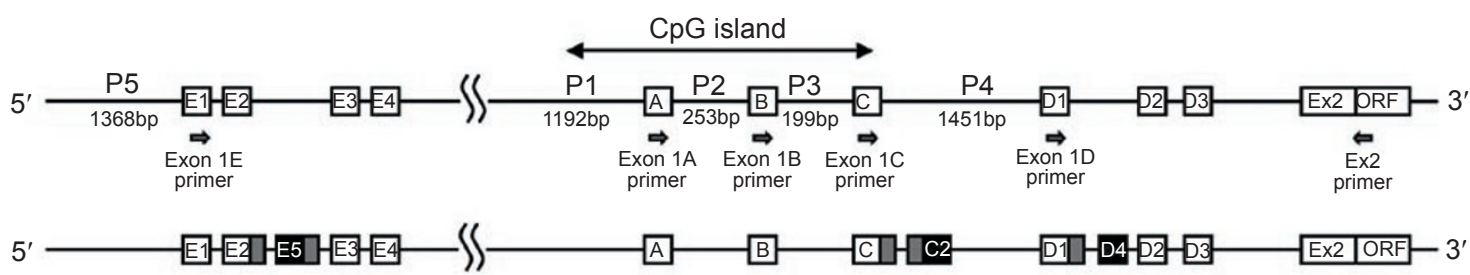

B

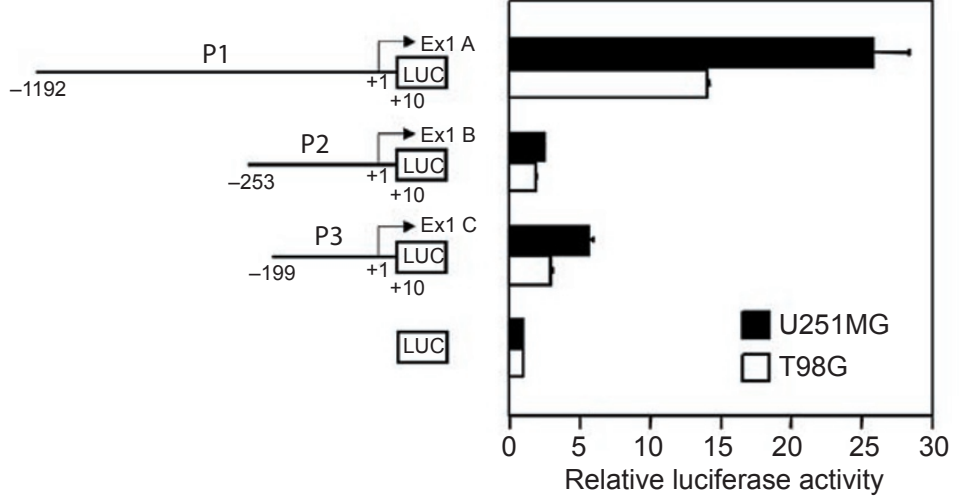

Figure 2 Activity of proximal CD133 promoters P1, P2, and P3 in glioblastoma cells. (A) Schematic representation of the newly identified exon-intron alignment of the human CD133 5'-UTR. Primers used for exon 1 analysis in Figure $1 \mathrm{~A}$ are indicated by white arrows. Upper and lower schemes represent the previously reported and newly modified genomic information for the human CD133 5'-UTR, respectively. Closed boxes represent the newly discovered exon $1 \mathrm{~s}$, and gray boxes represent the elongation of exons. The exons between 1E2 and 1E3, downstream of exon 1C, and between 1D1 and 1D2, were designated as exons1E5, 1C2, and 1D4, respectively. All splicing donor and acceptor sites conform to the GT-AG consensus rule. (B) Promoter activity of P1, P2, and P3 in U251MG and T98G glioblastoma cells. The sequences of three promoters registered in the GenBankTM/EMBL Data Bank were fused to the pGL3 enhancer vector, and their reporter constructs were transfected into U251MG and T98G cells, as described in Materials and Methods. The promoter activity of each transfection was expressed as the ratio of luciferase activity (firefly/renilla) and then normalized to the activity of the empty vector as 1 . Data are means \pm s.d. of values from three independent experiments. +1 indicates the transcription start position of each exon 1 . 
A
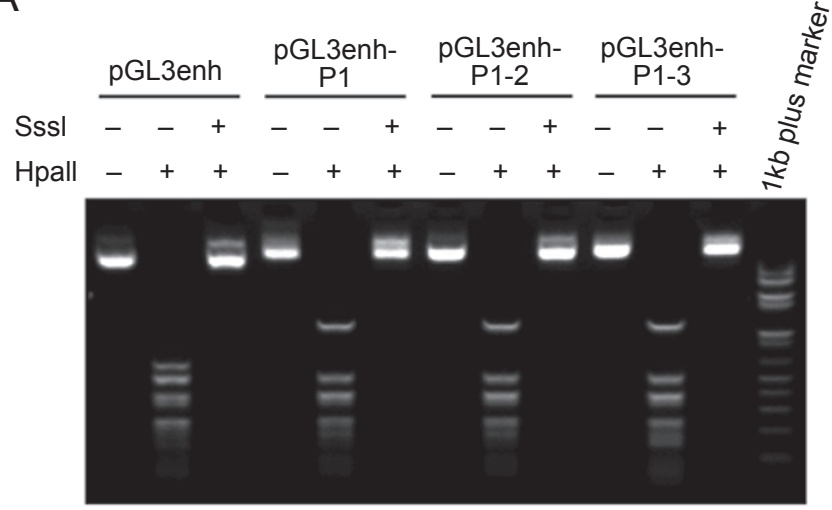

B
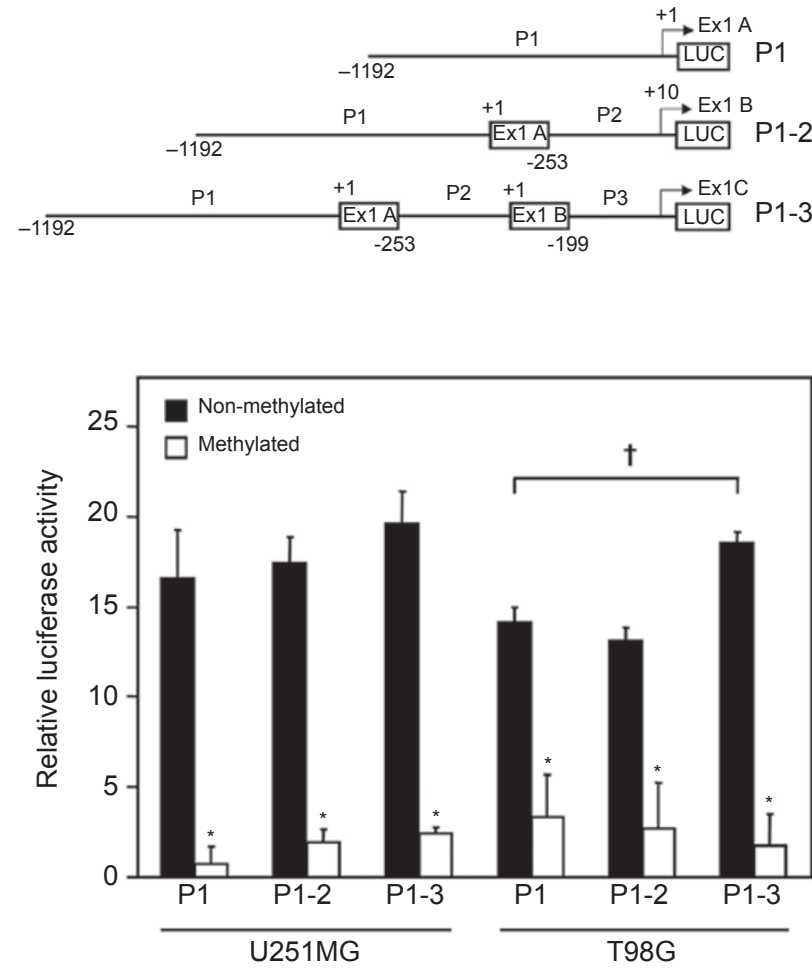

Figure 3 Synergic effect of the adjacent P2-P3 region and suppression by in vitro methylation on $\mathrm{P} 1$ activity in glioblastoma cells. (A) The digestion patterns of methylated and non-methylated reporter constructs using the methylation-sensitive restriction enzyme Hpall. The luciferase reporter constructs that contain the indicated CD133 promoter were methylated in vitro with SssI methylase, or they were not treated and thus remained unmethylated. Subsequently, each construct was digested with Hpall and electrophoresed. The molecular weight marker is also shown. (B) U251MG and T98G cells were transiently transfected with the methylated or unmethylated reporter constructs. Promoter activity of each transfection was expressed as the ratio of firefly/Renilla activity and then normalized to the activity of the methylated or non-methylated pGL3 enhancer empty vector as $1 .{ }^{\dagger} p<0.05$ vs P1. ${ }^{*} p<0.01$ vs non-methylated. Data are means \pm s.d. of values from three independent experiments. observed, while no significant enhancement of P2 (about 2.5-fold and 2-fold, respectively) and P3 (about 5.5-fold and 3-fold, respectively) was detected (Figure 3A). Similar results were obtained in a colon carcinoma cell line Caco2, which exhibited a comparable level of CD133 expression with glioblastoma tissues such as T18 and T20, for mRNA containing exons 1A, 1B, and 1C (Supplementary information, Figure S3A and S3B). In addition, we found that a synovial sarcoma cell line, Fuji, also exhibits an expression pattern similar to that of glioblastoma tissues, containing exons $1 \mathrm{~A}$ and $1 \mathrm{~B}$ of CD133 transcripts. This sarcoma cell line showed a modest increase in activity of the $\mathrm{P} 1$ promoter, and the activity of $\mathrm{P} 3$ was also increased. Therefore, transcripts of CD133 that contain exon 1A, 1B, or $1 \mathrm{C}$ and are present in glioblastoma tissues were thought to be driven primarily by promoter $\mathrm{P} 1$.

As described above, the $\mathrm{P} 2$ or $\mathrm{P} 3$ promoter alone did not exhibit significant activity in glioblastoma cells. To address whether $\mathrm{P} 2$ or $\mathrm{P} 3$ synergistically affects the activity of $\mathrm{P} 1$, we constructed reporter plasmids driven by a combined promoter of $\mathrm{P} 1$ and $\mathrm{P} 2$, or of $\mathrm{P} 1, \mathrm{P} 2$, and $\mathrm{P} 3$ (Figure 3B upper panel). Using our reporter assay, the activity of P1-2 was of nearly the same as that of P1 alone, in both U251MG and T98G cell lines. However, the activity of the P1-3 promoter was found to be modestly increased compared with that of $\mathrm{P} 1$ alone in the T98G cells (Figure 3B lower panel). In Caco-2 cells, the activity of the P1-3 promoter was found to be somewhat increased over that of P1-2, while the activity of P1-2 was nearly the same

A
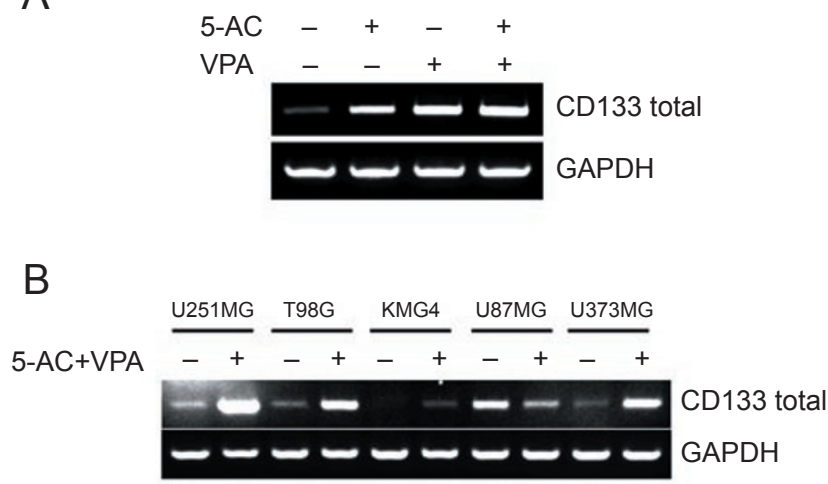

Figure 4 Reactivation of the $C D 133$ gene by epigenetic agents in various glioma cell lines. (A) Effects of the demethylating agent and the HDAC inhibitor on CD133 mRNA expression in U251MG. Cells were treated with $2 \mu \mathrm{M}$ 5-AC, 1 mM VPA, or a combination of 5-AC and VPA. RNA was reverse-transcribed, and expression of CD133 mRNA was analyzed by RT-PCR using 35 cycles. 5$A C, 5$-azacytidine as demethylating agent; VPA, valproic acid as a histone deacetylase (HDAC) inhibitor. (B) Effect of 5-AC in combination with VPA on CD133 mRNA expression in various glioma cell lines. 
magnitude as that of the $\mathrm{P} 1$ promoter alone (Supplementary information, Figure S3C). In Fuji cells, the activity of the P1-3 promoter was also found to be significantly increased. Thus, the $\mathrm{P} 2-3$ region of the CD133 promoter may contain cis-acting elements that can affect the $\mathrm{P} 1$ promoter activity, albeit in a cell type-specific manner.

\section{Methylation of $C p G$ sites represses $C D 133$ promoter activity}

The $\mathrm{P} 2$ and $\mathrm{P} 3$ promoters did not possess much transcriptional activity, but, considering that there are substantial numbers of $\mathrm{CpG}$ sites in the $\mathrm{P} 1-\mathrm{P} 3$ region, epigenetic regulation in these regions may contribute to CD133 expression. Using a CpG Island Searcher Program (http://cpgislands. usc.edu), $54 \mathrm{CpG}$ sites were located, and the percentages of CpGs in P1, P2, and P3 were determined to be $50 \%, 68 \%$, and $69 \%$, respectively. Therefore, we examined whether the promoter activity of the P1-P3 regions was affected by in vitro methylation. The promoter constructs were methylated using SssI methylase, and complete methylation was verified by digestion with the methylation-sensitive restriction enzyme HpaII (Figure 3A). In a reporter assay, in vitro methylation almost completely cancelled the activity of the P1-P3 regions, compared with their unmethylated forms (Figure 3B lower panel). These results suggest that the methylation status of the $\mathrm{P} 1, \mathrm{P} 2$, and $\mathrm{P} 3$ regions is associated with regulation of CD133 expression.

\section{Reactivation of CD133 by 5-azacytidine and VPA treat- ment}

To determine whether promoter methylation could be further linked to the loss of CD133 gene expression, glioma cells that showed low expression of the $C D 133$ gene were treated with the demethylating agent 5 -azacytidine (5-AC). As shown in Figure 4A, expression of CD133 mRNA was successfully restored by treatment with 5-AC in U251MG cells. Notably, combined treatment with 5-AC and valproic acid (VPA) induced CD133 expression in a synergistic manner. Similar results were obtained for the other glioma cell lines, T98G, KMG4, and U373MG (Figure 4B). Together, these findings indicate that DNA methylation serves to suppress the transcription of CD133 in glioma cells.
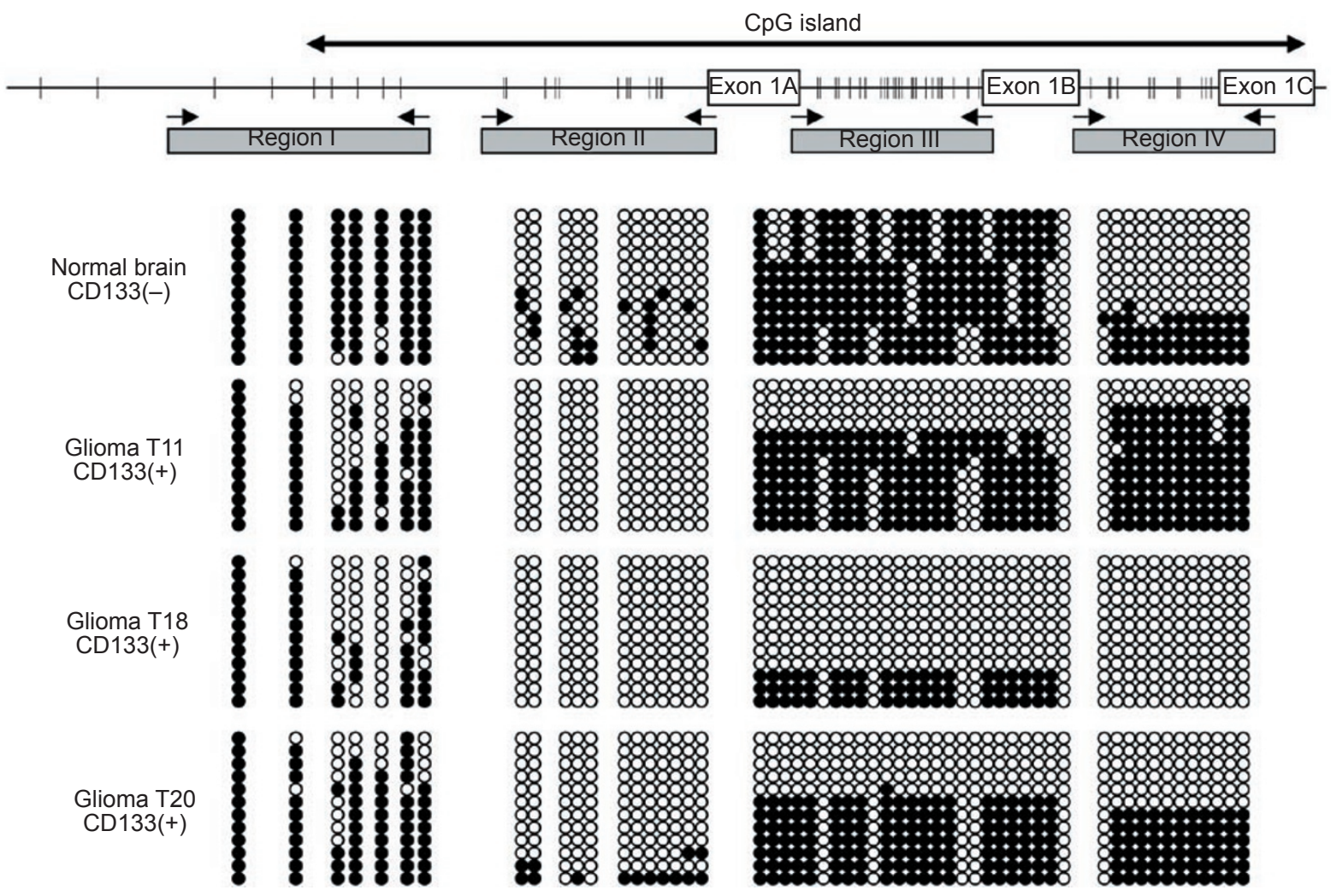

Figure 5 Methylation status of CpG sites within the P1-P3 region in normal brain and glioblastoma tissues. Mapping of methylated cytosines was carried out by bisulfite-treated genomic DNA sequencing. Vertical bars indicate CpG sites. The P1-P3 promoter region, including the $\mathrm{CpG}$ island, was divided into four regions, I-IV, defined by the PCR primers used to amplify the bisulfite-treated DNA. Twelve individual clones were analyzed per region and tissue sample. Closed circles represent methylated cytosines, and open circles represent unmethylated cytosines. 
Hypomethylation status and expression of CD133 in human glioma specimens

To determine the significance of promoter methylation in glioblastoma tissues, we performed bisulfite treatment and genomic sequencing of DNA from normal brain, lacking CD133 expression, and three cases of glioblastomas (T11, T18, and T20) with aberrant CD133 transcripts (Figure 5). The $\mathrm{P} 1-\mathrm{P} 3$ promoter regions, including the $\mathrm{CpG}$ island, were divided into four regions (designated as Regions I-IV), defined by the PCR primers used to amplify the bisulfite-treated DNA (Figure 5 upper scheme). Analysis of 12 clones per $\mathrm{CpG}$ site revealed that almost all of the CpGs in Region I were hypermethylated in the normal brain, while they were randomly demethylated in all examined glioblastomas. Region II also contained scattered methylation of $\mathrm{CpGs}$ in the normal brain, but all $\mathrm{CpGs}$ were completely demethylated in glioma cases T11 and T18. Dense hypermethylation of Region III was observed in normal brain, but all of the examined glioblastomas contained a proportion of completely demethylated alleles. Heterogeneous methylation in Region IV was also observed in all samples except glioma T18, which had completely demethylated CpGs. As glioblastomas comprise a heterogeneous population of CD133-positive and -negative cells (Figure 1B and 1C), these results clearly indicate that the $\mathrm{P} 1$ and $\mathrm{P} 2$ promoter regions tend to be hypomethylated in glioblastomas expressing CD133 mRNA, and that it is likely that a cluster of CD133-positive cells contain hypomethylated alleles.

\section{Discussion}

This study revealed the clinical significance of the presence of CD133 cells in glioblastoma and delineated a new regulatory mechanism of CD133 expression through epigenetic modification. These data provide new insights into the development of BTSCs defined by the expression of CD133 and will contribute to establishing effective therapies for glioblastoma.

We conclude that the expression of CD133 mRNA can be a strong diagnostic marker for malignant glioma. Among grade IV glioblastomas $71 \%$ (10 of 14) were shown to be positive for CD133 by RT-PCR, while in Grade I-III 75\% (6 of 8) were CD133-negative. RT-PCR analysis of CD133 expression was confirmed using immunohistochemistry (IHC) to examine tumor tissues. IHC revealed the focal presence of CD133-positive cells in micro-clusters. This localization may explain the possible false-negative results in RT-PCR, as sampling artifacts could lead to the exclusion of these CD133 micro-clusters. Thus, IHC analysis of serial tumor sections is critical for the estimation of CD133 expression levels, which are significantly involved in tumor malignancy. In addition, recent studies have reported a correlation between micro-clusters of CD133-positive cells in primary tumor tissues and patient prognosis [27]. As we could not obtain sufficient information on patient prognosis or tumor recurrence, further investigation is needed to determine the diagnostic value of CD133 expression in human glioma.

In elucidating the origins of gliomas and the basic mechanisms for the development of gliomas, it is critical to discriminate between NSCs and BTSCs, both of which harbor CD133. In this study, the expression of CD133s2 was found to be exclusively detected in glioma tissues but not in normal brain (Figure 1A panel ii). CD133s2 is known to be abundantly expressed in fetal brain, but is rarely found in adult brain [26]. Despite the fact that the functional differences between CD133s1 and CD133s2 are still unknown, our data strongly suggest that CD133s2 is a possible marker for distinguishing malignant BTSCs from normal NSCs. Some human prominin-1 splice variants, generated through alternative splicing of cytoplasmic C-terminal tails, have appeared in the NCBI GenBank database (accession numbers AY449690-AY449693) [28]. Thus, subsequent investigation of CD133 mRNA splicing variants might lead to the identification of a specific marker for human BTICs, which could help determine their cellular origin and malignancy.

Stem cell maintenance is known to be regulated by a specialized microenvironment (the so-called 'niche') composed of cell-extrinsic signaling molecules, such as Sonic hedgehog (Shh), Notch, and Wnt [29]. Therefore, the expression profiles of the downstream targets of these signaling molecules, including GLI1, HES1, and MYC, were also analyzed (Supplementary information, Figure S4). The expression pattern of HES1 was closely correlated with that of CD133, suggesting that CD133-positive cells in glioma tissue might be maintained by the Notch pathway, as previously reported for human medulloblastomas [30]. There was no significant correlation between expression of CD133 and EGFR or PDGFR $\alpha$, which are commonly amplified or overexpressed in human malignant gliomas [31].

This study also implicates the epigenetic machinery in the regulation of CD133 gene expression. In general, differentiated cells are predisposed to malignant transformation by the silencing of tumor suppressor genes through promoter methylation [23]. However, genomic hypomethylation has also been proposed to contribute to tumorigenesis by inducing the activation of oncogenes and/or genomic instability. For instance, DNA methyltransferase 1 (DNMT1) hypomorphic mice carrying genomic hypomethylation develop aggressive T-cell lymphomas, and the tumors typically display an increase in chromosomes [32]. Similarly, global hypomethylation thorough the induction 
of a hypomorphic allele of DNMT1 accelerates the onset of sarcoma formation in $\mathrm{Nfl}^{+/-} \mathrm{p} 53^{+/-}$mice [33]. Additionally, the deletion of DNMT1 in murine embryonic stem cells (ESCs) elevates mutation rates in both endogenous and integrated viral genes [34], and the disruption of DNMT1 and DNMT3b in the HCT116 colorectal cancer cell line results in constitutive genomic instability manifested by chromosomal translocations [35]. These studies indicate that DNA hypomethylation can cause genomic instability and initiate tumorigenesis. Thus, genomic hypomethylation might be associated with a susceptibility for developing and/or maintaining BTICs in brain tumors, resulting in the expression of the CD133 gene.

Demethylating drugs such as 5-AC have been used as anticancer agents because a variety of tumor suppressor genes have been shown to be silenced in human cancers by DNA hypermethylation [36]; however, in our study, treatment of 5-AC restored the transcription of CD133 in multiple glioma cell lines (Figure 4A and 4B). Interestingly, ESCs from Dnmt1-deficeint mice or from Dnmt3a/Dnmt3b double knockout mice proliferate as undifferentiated stem cells [37], indicating that the self-renewal capability is preserved in ESCs with hypomethylated genomes. In addition, these mutant ESCs fail to undergo differentiation in vitro, possibly owing to a failure to repress pluripotency-related genes such as Oct4 and Nanog [38]. As a reduction of methylation is thought to have the opposite effect on the proliferation of differentiated cells and on the self-renewal of stem cells, demethylating drugs might be effective for differentiated cancer cells but not for cancer stem cells. Therefore, treating glioma cells with conventional therapy followed by the selective DNA methylation of a stem cell-related gene may suppress the growth of any remaining BTICs.

In a portion of our experiments, we used serum-cultured glioma cell lines, which have been reported to undergo significant genomic alterations compared to primary gliomas [39]. Therefore, we confirmed our data using the human tumor cell lines Caco-2 and Fuji, which retain an expression pattern of CD133 mRNA that is similar to that of the glioblastomas, even in the serum-containing culture condition (Supplementary information, Figure S3). However, confirmation of our data using glioma stem cell lines would provide more information regarding the diverse mechanism of CD133 expression.

The expression of CD133 currently provides an important marker for various stem cell populations, including cancer stem cells. Nevertheless, it remains unclear whether CD133 influences stem cell-like properties, such as selfrenewal and differentiation. Alternatively, CD133 may only be a stem cell marker. It has been suggested that CD133 regulates cell migration and the interaction with the neighboring stem cell niche because it localizes to membrane protrusions [20]. The biological role of increased CD133 expression through promoter hypomethylation is undefined in the current study; however, because BTICs have enriched CD133 expression, epigenetic events including promoter hypomethylation might affect the development of human BTSCs.

In conclusion, this study elucidates the diagnostic significance of CD133 expression for glioma malignancy and the regulatory mechanism of its expression in glioma cells. Further studies of the mechanism underlying CD133 expression must be performed in order to understand the nature of CD133-positive BTICs and to develop future eradicative therapies.

\section{Materials and Methods}

\section{Glioma tissues}

Surgical specimens of brain tumors were obtained from patients who had undergone tumor resection at Iwamizawa Municipal General Hospital (Iwamizawa, Hokkaido, Japan) and Kashiwaba Neurosurgical Hospital (Sapporo, Hokkaido, Japan) between 2000 and 2007. Informed consent was obtained from all patients prior to their participation in the study. Portions of the tumor tissues were rapidly frozen by liquid nitrogen and stored at $80^{\circ} \mathrm{C}$ until RNA extraction.

\section{Cell culture, reagents, and transfection}

The U251MG, T98G, KMG4, U87MG, and U373MG glioblastoma cell lines, and Fuji synovialsarcoma cell line were cultured as described previously [40]. The human colon carcinoma cell line Caco-2 was kindly provided by Dr Masami Miyake (Research Institute for Microbial Diseases, Osaka, Japan) [41]. For reactivation of CD133 expression, cells were treated with $2 \mu \mathrm{M}$ 5-AC (Sigma) for an initial $48 \mathrm{~h}$ and then in combination with $1 \mathrm{mM}$ VPA (Sigma) for an additional $24 \mathrm{~h}$. Fugene HD transfection reagent (Roche, Indianapolis, USA) was used for transfection.

\section{Plasmids}

The pCR3.1-Uni-CD133s2 expression plasmid was kindly provided by Dr Denis Corbeil (BIOTEC, Dresden, Germany). To generate the $\mathrm{pGL} 3$ enh-P1, P2, P3, P1-2, and P1-3 reporter gene constructs, the promoter regions of CD133 were amplified by PCR using forward primers: 5'-ggtacc ACA ATT ATG GTG GAA GAA GGG AAA GC for P1, P1-2, and P1-3, 5'-ggtacc TCC CGC CGC GGT GAG TAT for P2, and 5'-ggtacc CGG GTG CAC GGT GAG TAG for P3 (a KpnI site is underlined), and reverse primers: 5'-ctcgag GTG GGG ATC TGC CTC AGT C for P1, 5' - ctcgag CCC TTC CCT TAG CTC GCC for P2 and P1-2, and 5'-ctcgag CAG TTC CTC TGG CCC CCA for P3 and P1-3 (a XhoI site is underlined), respectively. Genomic DNA isolated from normal human peripheral blood monocytes was used as a template. Oligonucleotide primers were synthesized using the published DNA sequence [GenBank accession number AY275524, nucleotides 700-2 459 for P1, P2, and P3]. The PCR products were gel-purified and cloned into pCR-Blunt II-TOPO vector (Invitrogen), verified by sequencing, and subcloned into the pGL3 enhancer luciferase reporter plasmid (pGL3enh) (Promega, Madison, WI, USA) with the $K p n I$ and $X h o I$ sites. 
$R N A$ extraction and semiquantitative $R T-P C R$

RNA was isolated from frozen tissues and cell lines with TRI Reagent (Sigma) according to the manufacturer's instructions. After conducting oligo(dT)-primed reverse transcription of $4 \mu \mathrm{g}$ of total RNA, the resulting single-stranded cDNA was amplified by PCR using KOD-Plus DNA polymerase (Toyobo, Osaka, Japan). Total RNAs from normal human kidney and testis were purchased from BD Biosciences Clontech (Palo Alto, CA). Primers are listed in Supplementary information, Table S4. Amplified products from exon 1 were cloned into pCR-Blunt II-TOPO vector and sequenced using the BigDye Terminator v1.1 Cycle Sequencing Kit (Applied Biosystems, Foster City, CA) on an ABI 310 genetic analyzer.

\section{Immunohistochemical analysis}

Formalin-fixed, paraffin-embedded tissues were used for immunohistochemical analysis according to the standard protocol. Briefly, for staining of CD133 without antigen retrieval, tissue sections were incubated with Protein Blocking Serum-Free (DAKO, Carpenteria, CA) for $10 \mathrm{~min}$ at $37^{\circ} \mathrm{C}$. Tissues were then incubated with an antiCD133 antibody (Abcam, Cambridge, UK) at $35 \times$ dilution overnight at room temperature. For nestin staining, an anti-nestin antibody (IBL, Japan) at $800 \times$ dilution was used after boiling in a pressure cooker for $2 \mathrm{~min}$.

\section{Luciferase reporter assay}

The dual-luciferase reporter assay was performed as described previously [42]. Briefly, cells plated in 24-well plates were transiently transfected with the CD133 promoter-firefly luciferase plasmid and the internal control plasmid pRL-TK (Promega), which encodes Renilla luciferase and is used to normalize transfection efficacy. The cells were incubated for $48 \mathrm{~h}$. All firefly luciferase activity was normalized to the Renilla luciferase activity, and data were presented as relative luciferase activity where control values were set to 1 for each cell line.

\section{In vitro DNA methylation}

In all, $4 \mu \mathrm{g}$ of CD133 promoter-based reporter plasmids, pGL3enh-P1, -P1-2, and -P1-3, were incubated for $4 \mathrm{~h}$ with 5 units of Sss ( $\mathrm{CpG}$ ) methylase (New England Biolabs, Beverly, MA) per $\mu \mathrm{g}$ of plasmid DNA. This reaction was performed in the presence (methylated) or absence (unmethylated) of $0.64 \mathrm{mM} S$-adenosylmethionine, as recommended by the manufacturer. After phenol extraction and ethanol precipitation, equal amounts of methylated and unmethylated reporter constructs were transiently transfected into cells, and luciferase activity was examined, as described above. Individual reactions were monitored by digestion with the methylation-sensitive restriction enzyme HpaII.

\section{Bisulfite-treated genomic DNA sequencing}

Genomic DNA was isolated from a normal cerebrum and three glioblastoma specimens with aberrant CD133 transcripts using the TRI Reagent according to the manufacturer's instructions. Bisulfite treatment was carried out following the modified procedure of Frommer et al. [43]. Briefly, $4 \mu \mathrm{g}$ of genomic DNA was digested with $K p n I$ and denatured with $0.3 \mathrm{M} \mathrm{NaOH}$ for $15 \mathrm{~min}$ at $37^{\circ} \mathrm{C}$. A freshly prepared solution of sodium bisulfite (3.0 M, pH 5.0) and hydroquinone $(500 \mu \mathrm{M})$ was added to the denatured DNA, and the mixture was incubated at $55^{\circ} \mathrm{C}$ for $4 \mathrm{~h}$. After desalting using the Wizard Genomic DNA Purification System (Promega), the DNA was desulfonated with $0.3 \mathrm{M} \mathrm{NaOH}$ for $15 \mathrm{~min}$ at $37^{\circ} \mathrm{C}$. Modified DNA was amplified by four sets of primers within the P1-3 regions. Primer sequences, locations, and PCR conditions are shown in Supplementary information, Table S4. All PCRs were performed using Qiagen Taq DNA Polymerase (Qiagen, Tokyo, Japan). PCR products were separated by $2 \%$ agarose gels, and bands were excised using the QIAquick Gel Extraction kit (Qiagen). Purified bands were cloned using a TOPO-TA cloning kit (Invitrogen). Twelve clones from each sample were sequenced.

\section{Statistical and computer analysis}

Comparisons between experimental groups were made using the Student's $t$-test with a significance level of $p<0.05$. The CpG island was predicted using the $\mathrm{CpG}$ Island Searcher Program (http://cpgislands.usc.edu).

\section{Acknowledgments}

We thank Dr Denis Corbeil (BIOTEC, Dresden, Germany) for pCR3.1-Uni-CD133s2 and Dr Masami Miyake (Research Institute for Microbial Diseases, Osaka, Japan) for Caco-2 cells. This study was supported in part by Grants-in-Aid from the Ministry of Education, Science, Culture, and Sports, Japan and from the Ministry of Health, Labor, and Welfare, Japan. Additional support was obtained from the YASUDA Medical Research Foundation, Japan; the Suhara Foundation, Japan; the Mochida Memorial Foundation for Medical and Pharmaceutical Research, Japan; and the UEHARA Medical Research Foundation, Japan. KT was supported by the YASUDA Memorial Medical Foundation.

\section{References}

1 Kleihues P, Louis DN, Scheithauer BW, et al. The WHO classification of tumors of the nervous system. $J$ Neuropathol Exp Neurol 2002; 61:215-225; discussion 226-219.

2 Maher EA, Furnari FB, Bachoo RM, et al. Malignant glioma: genetics and biology of a grave matter. Genes Dev 2001; 15:13111333.

3 Jordan CT, Guzman ML, Noble M. Cancer stem cells. N Engl J Med 2006; 355:1253-1261.

4 Singh SK, Clarke ID, Terasaki M, et al. Identification of a cancer stem cell in human brain tumors. Cancer Res 2003; 63:58215828.

5 Singh SK, Hawkins C, Clarke ID, et al. Identification of human brain tumour initiating cells. Nature 2004; 432:396-401.

6 Collins AT, Berry PA, Hyde C, Stower MJ, Maitland NJ. Prospective identification of tumorigenic prostate cancer stem cells. Cancer Res 2005; 65:10946-10951.

7 Ricci-Vitiani L, Lombardi DG, Pilozzi E, et al. Identification and expansion of human colon-cancer-initiating cells. Nature 2007; 445:111-115.

8 O'Brien CA, Pollett A, Gallinger S, Dick JE. A human colon cancer cell capable of initiating tumour growth in immunodeficient mice. Nature 2007; 445:106-110. 
9 Yin $\mathrm{S}, \mathrm{Li}$ J, Hu C, et al. CD133 positive hepatocellular carcinoma cells possess high capacity for tumorigenicity. Int J Cancer 2007; 120:1444-1450.

10 Monzani E, Facchetti F, Galmozzi E, et al. Melanoma contains CD133 and ABCG2 positive cells with enhanced tumourigenic potential. Eur J Cancer 2007; 43:935-946.

11 Olempska M, Eisenach PA, Ammerpohl O, et al. Detection of tumor stem cell markers in pancreatic carcinoma cell lines. Hepatobiliary Pancreat Dis Int 2007; 6:92-97.

12 Pfenninger CV, Roschupkina T, Hertwig F, et al. CD133 is not present on neurogenic astrocytes in the adult subventricular zone, but on embryonic neural stem cells, ependymal cells, and glioblastoma cells. Cancer Res 2007; 67:5727-5736.

13 Coskun V, Wu H, Blanchi B, et al. CD133+ neural stem cells in the ependyma of mammalian postnatal forebrain. Proc Natl Acad Sci USA 2008; 105:1026-1031.

14 Beier D, Hau P, Proescholdt M, et al. CD133(+) and CD133(-) glioblastoma-derived cancer stem cells show differential growth characteristics and molecular profiles. Cancer Res 2007; 67:4010-4015.

15 Dean M, Fojo T, Bates S. Tumour stem cells and drug resistance. Nature Rev 2005; 5:275-284.

16 Bao S, Wu Q, McLendon RE, et al. Glioma stem cells promote radioresistance by preferential activation of the DNA damage response. Nature 2006; 444:756-760.

17 Diehn M, Clarke MF. Cancer stem cells and radiotherapy: new insights into tumor radioresistance. J Natl Cancer Inst 2006; 98:1755-1757.

18 Liu G, Yuan X, Zeng Z, et al. Analysis of gene expression and chemoresistance of CD133+ cancer stem cells in glioblastoma. Mol Cancer 2006; 5:67.

19 Miraglia S, Godfrey W, Yin AH, et al. A novel five-transmembrane hematopoietic stem cell antigen: isolation, characterization, and molecular cloning. Blood 1997; 90:5013-5021.

20 Corbeil D, Roper K, Fargeas CA, Joester A, Huttner WB. Prominin: a story of cholesterol, plasma membrane protrusions and human pathology. Traffic (Copenhagen, Denmark) 2001; 2:8291.

21 Shmelkov SV, Jun L, St Clair R, et al. Alternative promoters regulate transcription of the gene that encodes stem cell surface protein AC133. Blood 2004; 103:2055-2061.

22 Feinberg AP, Tycko B. The history of cancer epigenetics. Nature Rev 2004; 4:143-153.

23 Baylin SB. DNA methylation and gene silencing in cancer. Nature Clin Pract 2005; 2(Suppl 1):S4-S11.

24 Ehrlich M. DNA methylation in cancer: too much, but also too little. Oncogene 2002; 21:5400-5413.

25 Cadieux B, Ching TT, VandenBerg SR, Costello JF. Genomewide hypomethylation in human glioblastomas associated with specific copy number alteration, methylenetetrahydrofolate reductase allele status, and increased proliferation. Cancer Res 2006; 66:8469-8476.

26 Yu Y, Flint A, Dvorin EL, Bischoff J. AC133-2, a novel isoform of human AC133 stem cell antigen. J Biol Chem 2002; 277:2071120716.

27 Zeppernick F, Ahmadi R, Campos B, et al. Stem cell marker CD133 affects clinical outcome in glioma patients. Clin Cancer Res 2008; 14:123-129.

28 Fargeas CA, Huttner WB, Corbeil D. Nomenclature of prominin-
1 (CD133) splice variants - an update. Tissue Antigens 2007; 69:602-606.

29 Shi Y, Sun G, Zhao C, Stewart R. Neural stem cell self-renewal. Crit Rev Oncol Hematol 2008; 63:43-53.

30 Fan X, Matsui W, Khaki L, et al. Notch pathway inhibition depletes stem-like cells and blocks engraftment in embryonal brain tumors. Cancer Res 2006; 66:7445-7452.

31 Fleming TP, Saxena A, Clark WC, et al. Amplification and/or overexpression of platelet-derived growth factor receptors and epidermal growth factor receptor in human glial tumors. Cancer Res 1992; 52:4550-4553.

32 Gaudet F, Hodgson JG, Eden A, et al. Induction of tumors in mice by genomic hypomethylation. Science (New York, NY) 2003; 300:489-492.

33 Eden A, Gaudet F, Waghmare A, Jaenisch R. Chromosomal instability and tumors promoted by DNA hypomethylation. Science (New York, NY) 2003; 300:455.

34 Chen RZ, Pettersson U, Beard C, Jackson-Grusby L, Jaenisch R. DNA hypomethylation leads to elevated mutation rates. Nature 1998; 395:89-93.

35 Karpf AR, Matsui S. Genetic disruption of cytosine DNA methyltransferase enzymes induces chromosomal instability in human cancer cells. Cancer Res 2005; 65:8635-8639.

36 Christman JK. 5-Azacytidine and 5-aza-2 -deoxycytidine as inhibitors of DNA methylation: mechanistic studies and their implications for cancer therapy. Oncogene 2002; 21:5483-5495.

37 Tsumura A, Hayakawa T, Kumaki Y, et al. Maintenance of selfrenewal ability of mouse embryonic stem cells in the absence of DNA methyltransferases Dnmt1, Dnmt3a and Dnmt3b. Genes Cells 2006; 11:805-814.

38 Jackson M, Krassowska A, Gilbert N, et al. Severe global DNA hypomethylation blocks differentiation and induces histone hyperacetylation in embryonic stem cells. Mol Cell Biol 2004; 24:8862-8871.

39 Lee J, Kotliarova S, Kotliarov Y, et al. Tumor stem cells derived from glioblastomas cultured in bFGF and EGF more closely mirror the phenotype and genotype of primary tumors than do serum-cultured cell lines. Cancer Cell 2006; 9:391-403.

40 Yao KC, Komata T, Kondo Y, et al. Molecular response of human glioblastoma multiforme cells to ionizing radiation: cell cycle arrest, modulation of the expression of cyclin-dependent kinase inhibitors, and autophagy. J. Neurosurg 2003; 98:378-384.

41 Miyake M, Hanajima M, Matsuzawa T, et al. Binding of intimin with Tir on the bacterial surface is prerequisite for the barrier disruption induced by enteropathogenic Escherichia coli. Biochem Biophys Res Commun 2005; 337:922-927.

42 Tabu K, Ohnishi A, Sunden Y, et al. A novel function of OLIG2 to suppress human glial tumor cell growth via p27Kip1 transactivation. J. Cell Sci 2006; 119(Part 7):1433-1441.

43 Frommer M, McDonald LE, Millar DS, et al. A genomic sequencing protocol that yields a positive display of 5-methylcytosine residues in individual DNA strands. Proc Natl Acad Sci USA 1992; 89:1827-1831.

44 Colella S, Ohgaki H, Ruediger R, et al. Reduced expression of the Aalpha subunit of protein phosphatase $2 \mathrm{~A}$ in human gliomas in the absence of mutations in the Aalpha and Abeta subunit genes. Int J Cancer 2001; 93:798-804.

(Supplementary information is linked to the online version of the paper on the Cell Research website.) 